\title{
Enhanced AODV for Mobile Ad-hoc Networks
}

\author{
Rohit Sharma \\ Department of CSE \& IT \\ Lovely Professional University, India
}

\author{
Sangeeta Sharma \\ Department of CSE \& IT \\ Lovely Professional University, India
}

\begin{abstract}
A mobile ad-hoc network comprises of mobile nodes that can communicate with the nodes within their radio range and also with those which are outside range by using route discovery mechanisms. The nodes in an ad-hoc network can act as a host and also as a router that can route data to or from the other nodes. The topology of mobile ad-hoc networks changes dynamically which is mainly due to arrival or departure of nodes from the network. The nodes in ad-hoc network are self-adjusting and can adapt to the changes occurred in the topology. The main limitation of mobile ad-hoc networks is the energy constraints. There are three states of a node that results in energy consumption that are transmitting, receiving and idle state. The energy consumption of a node leads it to get down and makes it out of the network. This will lead to change in topology which incurs routing overhead when information about the down node has to be transferred to each node in the network. Again it will result in consumption of energy which incurs due to processing of these packets containing the information about the down node. In this paper we are presenting an enhanced on demand routing protocol in which each node uses a threshold value for energy and when the value of energy of that node reaches the threshold that node will not participate in the data transfer and when all the nodes in the network possess the set threshold value the threshold value is decreased. The enhanced protocol is based on Ad-hoc On Demand Distance vector (AODV) and hence named as Enhanced AODV (EAODV). In this paper the working of the EAODV is explained and also the simulation of the protocol is shown using the NS2 simulator. The paper also shows how this protocol is better than simple AODV based on different performance factors. The EAODV mainly concentrates on using the energy of nodes in an efficient manner so that the lifetime of a network can be increased.
\end{abstract}

\section{Keywords}

AODV, EAODV, Mobile Ad-hoc Network, Threshold.

\section{INTRODUCTION}

A Mobile ad-hoc network consists of mobile nodes which forms a network independent of any standalone infrastructure. The nodes in the network can directly with the nodes within its range and can indirectly communicate with all other nodes suing some route discovery mechanism. MANETs can be used in tactical communications such as military operations, personal area networks, disaster managements and in wireless sensor networks too. The figure shows an example of a Mobile ad-hoc network in military operations:

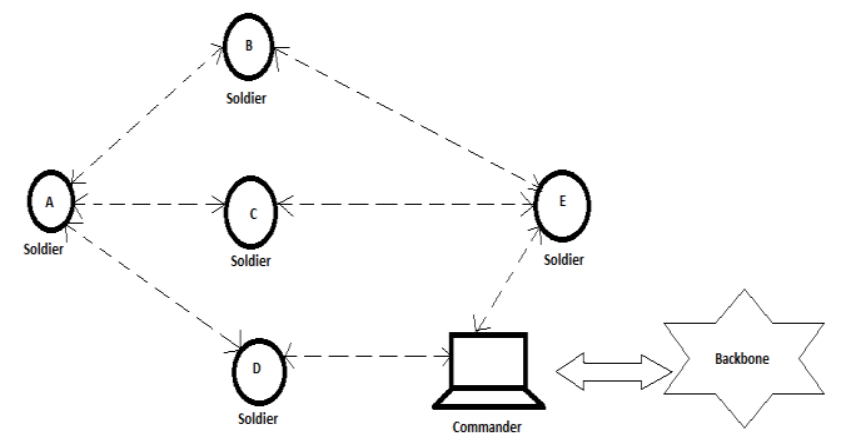

Figure1: Mobile Ad-hoc Network used in military operation

Ad-hoc networks are multi-hop networks in which all the nodes cooperatively maintain network connectivity. These networks are very useful where there is need to establish a temporary network for a short span of time such as in military or disaster management operations. In such networks, designing energy-efficient routing protocols is very important as the nodes have only limited amount of energy.

Recent studies have stressed the need for designing protocols to ensure longer battery life. The nodes remain powered most of the time even when they do not have any sought of work to do. The useful energy of nodes is wasted in overhearing other transmissions whether those transmissions are related to them or not. The most of the energy got wasted in updating the routing information when the topology of the network changes. The life a network solely depends upon the lifetime of the nodes in the network. The nodes should be efficiently used so that the nodes do not run out of the energy before the entire process has been completed. The advancement of mobile computing and communication devices is driving a revolutionary change in our information society. Now we can access whatever information we require from anywhere and from any part of the world. The latest technologies and using devices that supports such features of remotely connecting to a network and accessing any kind of information has made wireless networks the easiest solution for their interconnection. In today's scenario now mobile users can also use their cellular phone to check the e-mail, browse internet and travelers with portable computers can access the internet from airports, railway stations and from other public locations where there is facility to connect to the network, tourists can use Global Positioning System (GPS) terminals installed inside the cars and now in mobiles to locate driving maps and tourist attractions [1]. Using this technology people can exchange files and other information by connecting portable computers via wireless LANs during conferences from homes. The users can also synchronize their data and transfer files between portable devices and desktops. The MANET also permits the multipath routing which enables formation of multiple paths between source nodes to 
destination node. This is proposed in order to enhance the reliability of data transmission and to provide load balancing feature. The MANET is also characterized by variablecapacity, bandwidth-constrained wireless links and dynamic changes in topology which leads to frequent changes in connectivity which are unpredictable too. A major challenge that exists in ad hoc networks is that if a host does not communicate on its own but it still keeps on forwards data and routing packets for others which results in drain of its battery. There are basically the three states where the energy is consumed during the life of a node: 1). during transmitting the data, 2) During receiving the data and, 3) During the period when the node is idle. The first two states are necessary part during the communication but the consumption of energy during the idle state is just the waste of energy. One of the ideas thus evaluated to conserve such a waste of energy is to switch off a non communicating node for the time period until it is not required in some communication. This idea off switching off a node to conserve battery power may not be always a good idea because it can partition a network. The Conventional on-demand routing protocols such as AODV and DSR are energy-unaware routing protocols [2]. In these protocols routing is done based on shortest path or by calculating the cost metric during the establishment of the route. The routes are not proactively modified in these routing protocols until the existing routes breaks down.

Our main focus is on augmenting the existing on-demand routing protocols and making them energy efficient. The On demand protocols are suitable for this study as they typically have lower routing overhead than proactive protocols and have a low baseline consumption of energy. The protocol that is discussed here is AODV and the techniques that we have applied over AODV can be applied to other on demand routing protocols also. The main aim of the paper is to make AODV more energy efficient by efficiently using the energy of the nodes in the network and not allowing the network to get divided resulting in long lifetime of the network. In the paper AODV and Enhanced AODV (EAODV) is also compared on various performance factors showing the changes that come in the performance of AODV after the techniques we applied on it.

\subsection{MANET Routing Protocols}

The Ad-hoc networks are mobile wireless networks in which the infrastructure is not fixed. There are no fixed routers as each node acts as a router and forwards traffic from other nodes [3]. The MANET has various routing protocols some of which are shown in the figure below. The routing protocols are divided into two categories i.e. Proactive and Reactive Routing Protocols these protocols are also discussed in the next sub section.

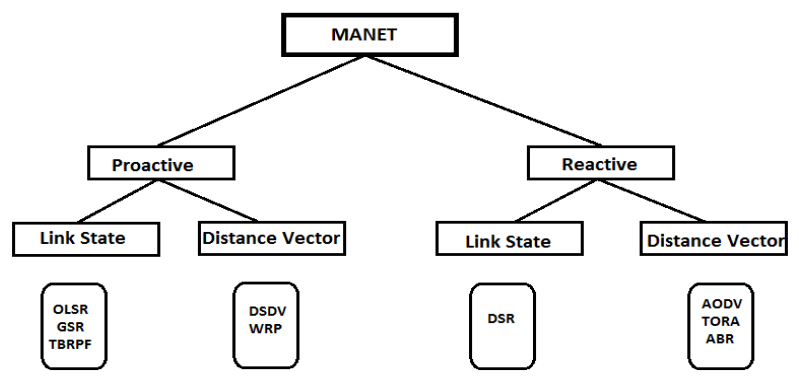

Figure2: Routing Protocols in MANET

\subsubsection{Proactive Routing Protocols}

The Proactive routing protocols maintains one or more routing tables for nodes in the network. In proactive routing protocols there is always a route available from one node to all other nodes in the network. The routing table is updated periodically in these protocols and also when the topology of the network incurs some changes. In these protocols the source node does not require any route-discovery procedure to search a route to other node in the network. Proactive protocols evaluates the routes within the network continuously so that when we are required to forward the packet route is already known and immediately ready for use [4]. There is no time delay takes place. The examples of Various Table Driven protocols are: Cluster gateway switch routing (CGSR), DSDV, Fish eye state routing (FSR), Optimized link state routing (OLSR), etc.

\subsubsection{Reactive Routing Protocols}

The Reactive protocols are also known as on demand protocols which have no network information at nodes if there is no communication currently in process. The Reactive protocols do not maintain or constantly update their route tables with the latest route topology. A node when wants to send a packet to another node then this protocols searches for the route and establishes the connection in order to complete the data transfer. Reactive Routing Protocols are more efficient than proactive routing and most of the current work and modifications have been done in this type of routing. Mechanism used in reactive protocols for routing is to search a route between a source and destination only when a communication has to undergo [5]. Discovery of route on demand avoids the cost of maintaining routes and controls the traffic of the network. The examples for on demand protocols are: ad-hoc on demand distance vector (AODV), Dynamic source routing (DSR).

\subsection{Routing Approaches}

The Reactive Routing Protocols and Proactive Routing Protocols use two routing techniques that are followed by its protocols those are: Link State Routing and Distance Vector Routing. These both techniques are discussed below:

\subsubsection{Link State Routing}

The link state routing technique is used both by reactive as well proactive routing protocols. The link state routing is used in packet switching networks for communication purposes. The examples of link state routing protocols are DSR, OLSR, GSR etc. the link state routing is performed by every switching node in the network. The concept behind the mink state routing is that every node in the network constructs a map of the topology of the network [6]. The network topology is thus mapped is in the form of a graph which shows which node is connected to which node of the network. Each node in the network calculates the next best logical path that results are stored in the routing table. This approach differs from distance vector in the sense that in it each node shares its routing information with its neighbors. The information that is passed to the neighbors is just connectivity related.

\subsubsection{Distance Vector Routing}

The Distance Vector Routing does not have knowledge of the entire path from sender to the receiver. The two method that distance vector uses are: The direction in which router or exit interface a packet should be forwarded and Distance from the destination. Distance Vector Protocols are based on the calculation of the direction and distance to any link in a network. By the direction it means the next hop address and 
the exit interface. It is basically a measure of the cost to reach a destination from the sending node. Lesser be the cost between two nodes means the shortest path between them. In Distance Vector Routing each node maintains a vector of minimum distance to every node that is also called as vector table. The cost to reach a destination depends on the various route metrics. For example, RIP uses the hop count of the destination whereas IGRP takes into account other information such as node delay and available bandwidth etc. In Distance Vector Routing updates are performed periodically where all or part of a router's routing table is sent to all its neighbors. RIP supports cross-platform distance vector routing whereas IGRP is a Cisco Systems proprietary distance vector routing protocol. When a router has this information it is able to add its own routing table to reflect the changes and then inform about the changes to its neighbors. The router relies on the information they receive from other routers and cannot determine cannot able to determine whether the information is valid or invalid [7]. The various examples of the protocols that use this approach are: - DSDV, WRP, TORA, ABR, etc.

\section{AODV Protocol Description}

AODV uses an on demand approach for finding the routes i.e. a route is established only when it is required for transmission of data. It makes use of the destination sequence numbers to identify the most recent path. The source node in AODV maintains the next-hop information corresponding to each flow for data transmission. In AODV, the source node floods the Route Request packet to the network [8]. Each node on receiving the route request packet forwards it to its neighbor nodes. Similarly each node forwards this packet until it reaches the destination. On receiving the Route request packet destination replies using a Route reply packet and then a route is selected on the basis of hop-count and if the routes contains same number of hop counts a random path is taken. The whole process is explained below:

\subsection{Route Request (RREQ)}

In AODV whenever the source node wants to communicate with other node in the network a route is established. The Source node broadcasts a route request packet RREQ to its neighbor nodes. On receiving the RREQ form source the nodes again broadcasts the RREQ to their neighbors. Following the same procedure finally the RREQ reaches the destination. A RREQ carries the Source identifier (SrcID), destination Identifier (DestID), the source sequence number (SrcSeqNum), the destination sequence number (DestSeqNum), the broadcast identifier (BcastID), and the time to live field (TTL) which specifies the lifetime of the RREQ. The destination sequence number indicates the freshness of the route. The RREQ can be received multiple times for this Broadcast ID is maintained so that the similar RREQ can be discarded [9].

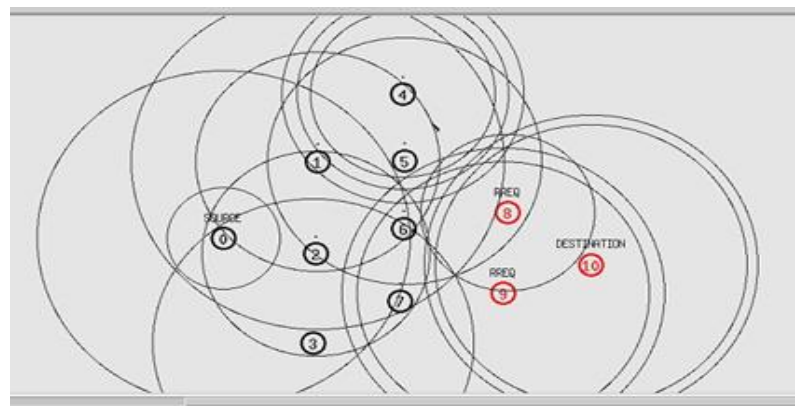

Figure3: RREQ reaches the Destination Node

\subsection{Route Reply (RREP)}

When the RREQ reaches the destination it replies with a route reply packet (RREQ). The destination can receive the RREQ from different nodes so it broadcasts the RREP to the neighbor nodes. This will lead to source getting the multiple routes. The intermediate nodes on receiving RREP updates their routing tables with the latest destination sequence number. Source on receiving multiple routes selects the route on the basis of the hop count. The source will select the route with less number of hop counts [10]. If the two paths contain the same path it will choose a random path. The figure below shows a RREP packet sent to node 8 and 9 by the destination:

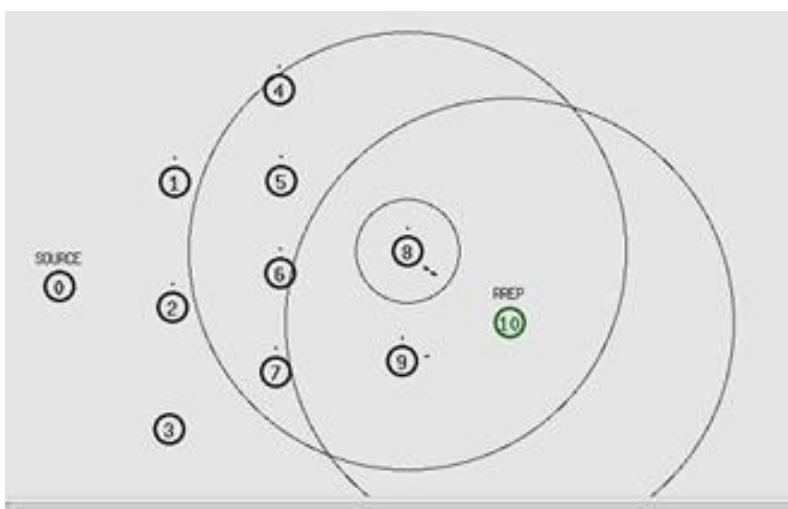

Figure4: Destination sends the RREP

\subsection{Route Establishment}

The figure below the route thus established between the source node and the destination. The nodes that became the part of the route are shown blue in color. Now this route is followed for the communication between source and the destination.

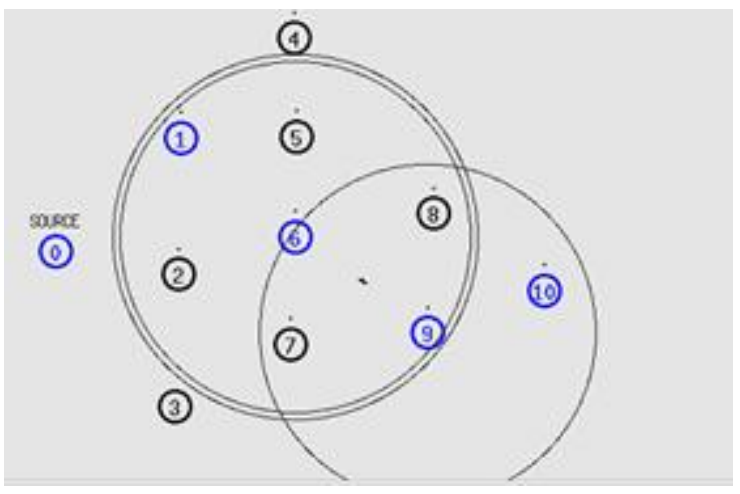

Figure5: Route Establishment

The above section explained the complete working of the AODV protocol. One of the main issues that are with AODV is that it does not repair the broken paths. When a link break occurs, the source node learns about it and route reestablishments have been done.

\section{Enhanced AODV}

EAODV uses the same mechanism as of AODV. The source node sends the RREQ when it wants to communicate with the other node in the network. The route establishment procedure is same as AODV. On receiving the RREQ the destination replies with RREP packet and when RREP reaches the source a path has been selected. EAODV differs from AODV as in EAODV in RREP each node sends its energy level to the source node. On receiving the RREP the source node selects the route based on hop count and the energy of the nodes in 
that route. EAODV uses the energy threshold value concept in which every node in the network has minimum energy level set to $25 \%$ i.e. the energy threshold value for each node is set to $25 \%$. Whenever a node energy level reaches $25 \%$ that node will not take part in the communication. Then a new path will be taken which is best available at that time. As in AODV for establishing a route when a route node in fails can result in division of network or change in path again leads to routing overhead results in more energy consumption. But in EAODV no such things happens. Each node in EAODV maintains the energy level of the nodes in the network as routing information, whenever a node reaches threshold value a new node takes its place. The new route taken in EAODV does consume energy and does not produce routing overhead. When all the nodes in the network reaches the threshold value or the nodes 1, 2, 3 as shown in figure below becomes $25 \%$ then there is no other route left for data transfer, in this case the threshold value is set to $5 \%$.

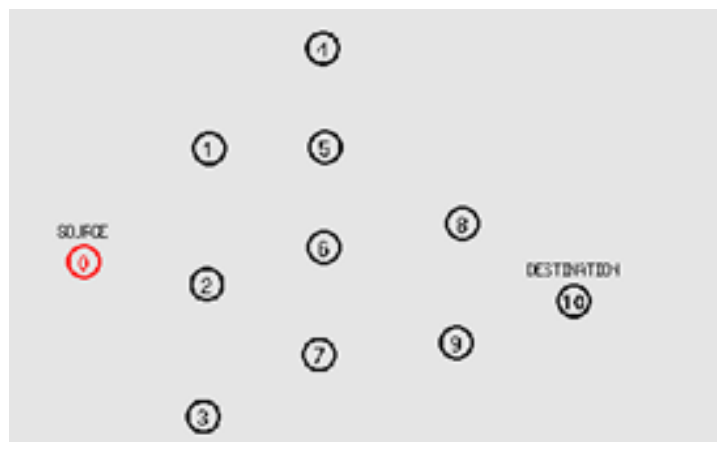

Figure6: EAODV Scenario

\subsection{RREQ, RREP and Route}

\section{Establishment in EAODV}

The route establishment concept of EAODV is same as AODV the difference is simply in RREQ. In EAODV the energy level of each node is appended in the RREP. Thus the source node will know the energy of each node in the network and chooses the route for communication on the basis of hop count and the energy level of nodes that would be the part of the route. The following figure shows the RREP in EAODV each node send its energy level in RREP to their neighbor nodes:

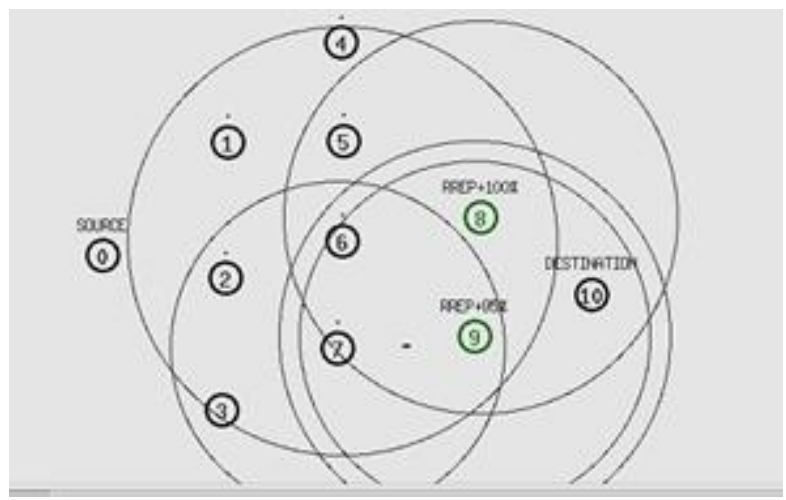

Figure7: RREP in EAODV

\subsection{Threshold Value Concept}

EAODV uses threshold value for energy which is set to $25 \%$ in our implementation. Each node in the network possesses the threshold value set to $25 \%$. Whenever the value of a node reaches $25 \%$ it will not take part in the communication hence the other node in the network takes its place and the communication keeps going. The threshold value is represented as Th and energy is represented as E. the equation thus formed is when energy $\mathrm{E}$ of node becomes less than or equal to $\mathrm{Th}$ that node does not take part in the communication:

$$
\mathrm{E}<=\mathrm{Th}
$$

Here $\mathrm{Th}=25 \%$

The following figure shows this approach:

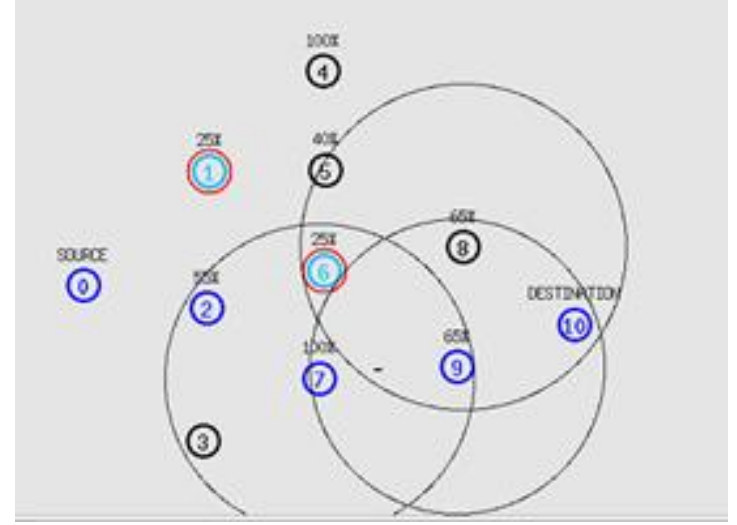

Figure8: Threshold Value Concept

Here in the above figure the node 1 Th value reaches threshold value and hence the node 2 is included in the route. Similarly after some time the node 6 value also reaches Th value So the node 7 is included in the path.

\subsection{Re-setting the Threshold value}

Re-setting the Th value is another advantageous approach in EAODV. When all the nodes in the network possesses the value equal to or less than the set Th value the Th value is again reset to 5\%. Again the protocol works as same as mentioned above.

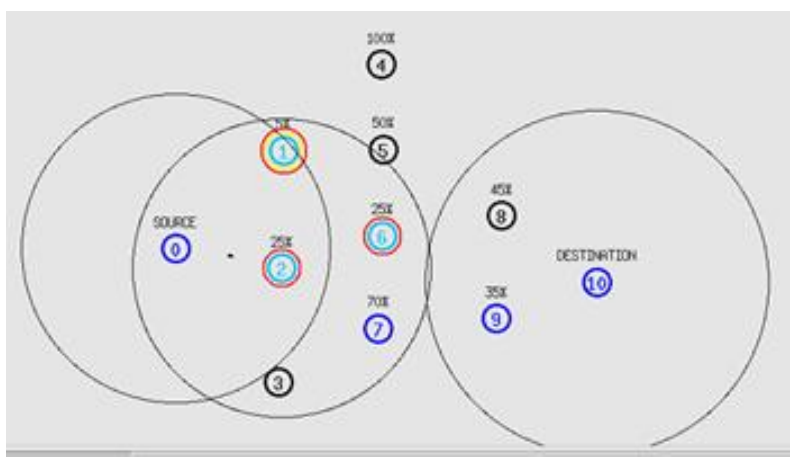

Figure9: Re-setting the Threshold value

The figure above shows the re-setting of Th value. There exists another case as shown in figure that can result in resetting the Th value. Suppose if nodes 2 and 3 both reaches the Th value $25 \%$ and node 3 is not in range of source node then the communication will halt in such cases EAODV adapt to this situation and reset the values of threshold to $5 \%$.

\section{SIMULATION AND PERFORMANCE METRICS}

\subsection{Simulation Tool}

The simulation tool that we are using is Network Simulator NS2. NS is an event driven network simulator developed at UC Berkeley that can simulates variety of IP networks. The implementation of network protocols such as TCP, UPD, FTP, Telnet, Web, CBR and VBR can be done in NS2. The simulation scripts in NS2 are developed using the tool 
command language (Tcl). NS2 supports for simulation over wired and wireless networks such as TCP, routing, and multicast protocols. NS2 is object oriented simulator and its code is written in C++ and the frontend to be used is OTcl interpreter. So as to develop components for ns 2 both the Tcl and $\mathrm{C}++$ have to be used. The most of the simulation scripts are developed in Tool Command Language (Tcl) [11]. The figure below shows the basic view of NS:

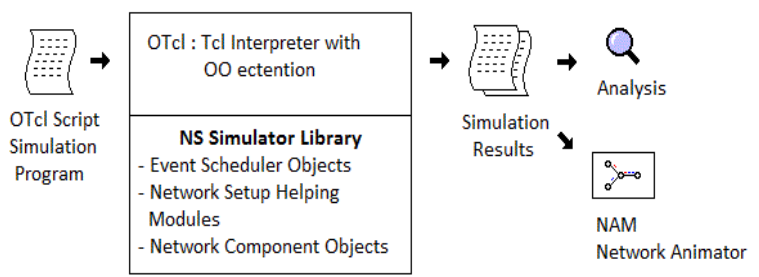

Figure10: NS User View

\subsection{Performance Metrics}

The AODV and EAODV reactive routing protocols will be evaluated on the basis of the following factors:-

\subsubsection{Average End to end delay of data packets}

Average end to end delay is measure of the average time from the beginning of a packet transmission at a source node until packet reaches the destination. These delays are caused by buffering of data packets which incurs during discovery of routes. The cause for delay is the queuing that occurs at the interface queue. The measurement also includes retransmission delays and also the propagation times. For the calculation of delay the sending $(\mathrm{S})$ time $(\mathrm{t})$ and receiving $(\mathrm{R})$ time $(\mathrm{T})$ and then we calculate the average. The information thus we get will help in plotting the graph which shows which protocol is better.

\subsubsection{Throughput}

The throughput is the measure of total number of packets successfully delivered to the destination. More the throughput more the protocol is efficient for transmission. Here AODV and EAODV will be compared on the basis of throughput.

\subsubsection{Packet Delivery Fraction}

The packet delivery fraction is the ratio of the number of data packets that is transmitted using CBR sources successfully delivered to the receiver. The calculation for packet delivery fraction can be performed using the following formula:

Packet delivery fraction $=$ (Received packets/Sent packets)*100

\subsubsection{Energy Consumption}

Energy is a major constrain in mobile ad-hoc networks. A critical issue during routing is to conserve as much power as we can so as to achieve high throughput. In this performance metric we will see the changes that we made to AODV by applying our technique will be able to yield better results [12].

\section{RESULTS}

\subsection{Average End to End Delivery:}

The following figure shows the average end to end delay between AODV and EAODV. As we know that that average end to end delay occurs due to discovery of nodes and also due to transmission delays, the figure shows that the EAODV possess less end to end delay as compared to AODV. The green curve in the graph shows the curve for AODV and red curve shows the curve for EAODV.

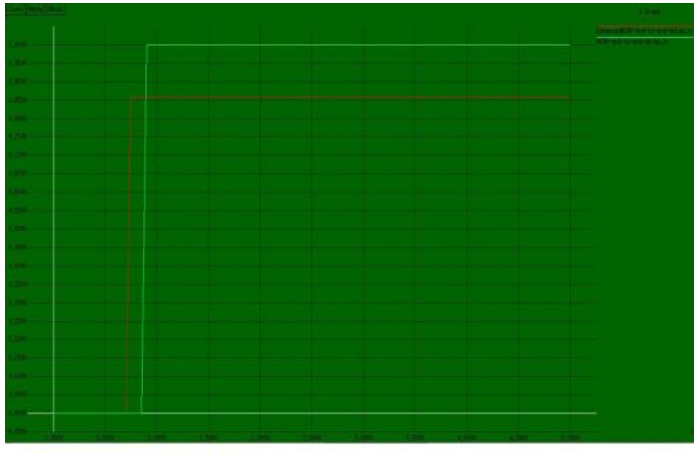

Figure11: Average End to End Delay

\subsection{Throughput:}

Throughput is the measure of total number of packets successfully delivered to the destination. The figure below shows the curves for throughput for AODV and EAODV. The green curve is of AODV and the red is for EAODV. As we can clearly see that after applying our approach EAODV has produced better output.
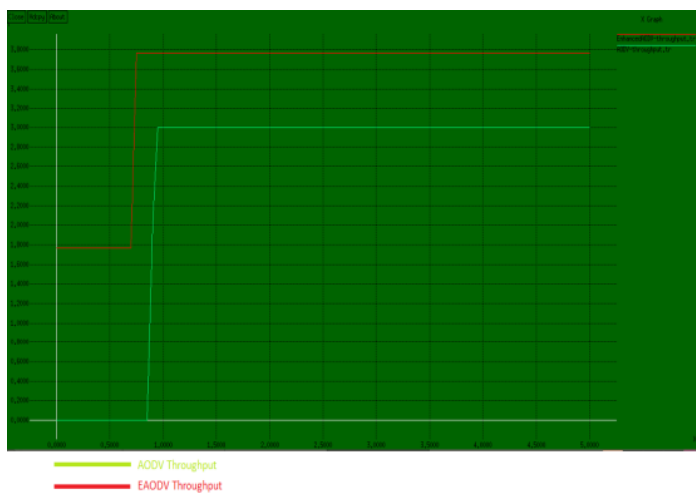

Figure12: Throughput

\subsection{Packet Delivery Fraction:}

The following figure shows the packet delivery fraction curves for AODV and EAODV. the measure is of ratio of the packets that are delivered to the destination that were sent using CBR to the total packets that were sent. The green curve is for AODV and Red is for EAODV. It is clear from the curves that EAODV yields better output than AODV.

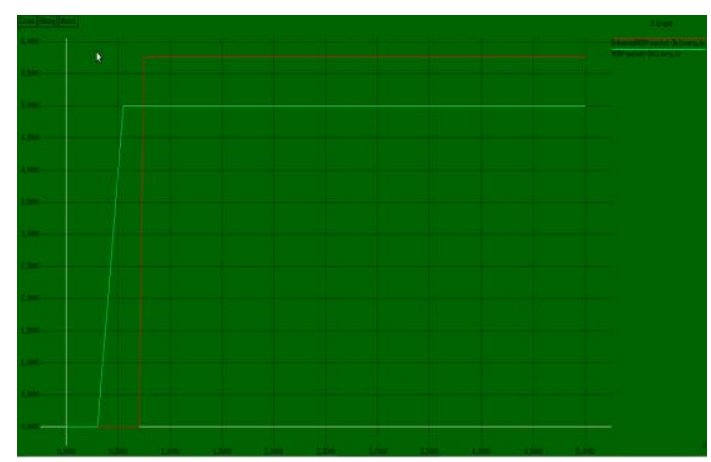

Figure13: Packet Delivery Fraction

\subsection{Energy Consumption:}

As discussed earlier energy is the biggest constraint in mobile ad-hoc networks. The main aim of our enhanced AODV is to efficiently use the energy of the nodes in the network so that 
lifetime of the network could be increased. The following figure shows the result that is produced in our simulation. The green curve shows the energy consumption of AODV and red curve shows the energy consumption for EAODV. The results shows that EAODV is energy efficient than AODV. The EAODV has provided better results and is helping in better utilization of energy.

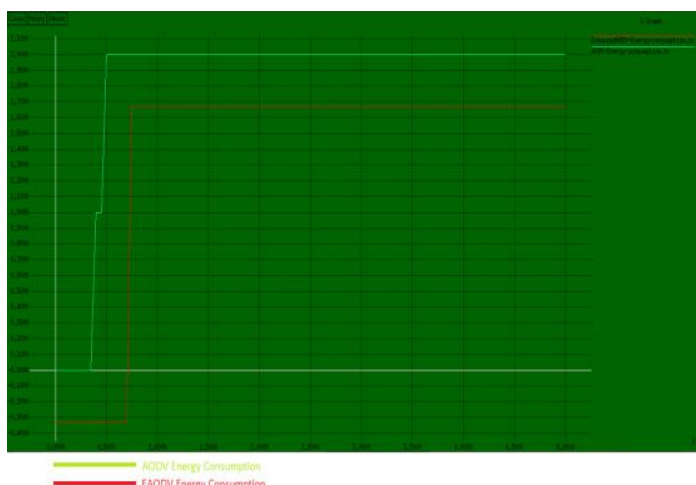

Figure14: Energy Consumption

\section{CONCLUSION \& FUTURE SCOPE}

The On demand routing protocols because of their low routing overhead are very useful for mobile ad hoc network environment. The energy is major concern in all sought of wireless communication. The approach used here in EAODV has proven that we can reduce energy consumption through various ways. The fixing of fixed threshold value for all the nodes has helped in fairly utilizing the energy of node in the network. The EAODV has increased the lifetime of the network to a great extent and also helped to prevent the network to get divided into different networks. Its capability to adapt itself according to the situation makes it more suitable for mobile ad-hoc environment. It changes its threshold value when all the nodes possess the threshold value and also alters the threshold value if the front nodes values reaches threshold and there is no other route to transfer data. The analysis of the AODV and EAODV on demand routing protocols had shown that EAODV performs better than AODV. EAODV has better Packet delivery fraction than AODV so therefore EAODV performs better where data has to be transferred at CBR. The end to end delay is also less for EAODV makes it better than AODV. EAODV has found solution to the energy issue and has performed better than AODV. The analysis has shown that EAODV is more energy efficient than AODV and its energy efficiency can be improved further using techniques like load balancing and also by reducing routing overheads. The throughput of EAODV is also better than AODV, the results has shown that with less losses takes place in EAODV the throughput is better. The changes in applications occur time to time and in this technique also improvements can be done to yield better results. The applications can be enhanced by adding some new features so that better and effective results could be gained. In order to take this work to a broader level some of the features that could be added to this work are:

1. Maintaining the interoperability with fixed infrastructure is a big challenge. The MANET is having autonomous capabilities and it oftentimes is desirable to connect it to fixed infrastructures. The designing of such an application includes major challenges like addressing, mobility management issues, security issues and transport layer functionalities.
2. The packet size that could be increased and then the both the protocols can be compared and the changes in the result could be analyzed. The packet size could be increased enough so that segmentation and reassembly should takes place at sender and receiver side respectively.

3. The application can be enhanced by increasing the number of the nodes. Increasing the number of nodes will take the application to another level and hence the changes in the result could be analyzed.

4. The efforts could be made to improve the address configuration in an ad-hoc network. Special care should be taken when node movement and network partitioning occurs.

5 . The efforts could be made to enhance make advancements in link layer and physical layer to enable low latency services over long distance.

\section{REFERENCES}

[1] Chlamtac Imrich, Conti Marco, Jeniffer J,-N, Liu (2003)" Mobile Ad-hoc Networking imperatives and challenges" School of engineering, University of Dallas, USA.

[2] Marco Fotino and Floriano De Rango "Energy Issues and Energy Aware Routing in Wireless Ad-hoc Networks" University of Calabria, Italy.

[3] Beijar Nicklas "Zone Routing Protocols" Networking Laboratory. Helsinki University of Technology, Finland.

[4] Chansu Yu, Ben Lee, Hee Youg Youn "Energy efficient routing protocols for MANET" Department of ECE, Cleveland state University, Oregon State University, Sungkyunkwan University, Korea.

[5] Sharma Rohit and Sharma Sangeeta, "Simulation and Performance Analysis of Reactive Routing Protocols" Lovely Professional University, India.

[6] Hao Jutao, ZhaoJingjing and Li Minglu "Energy Level and Link State Aware AODV Route Request Forwarding" Univedrsity of Shanghai, China.

[7] Corson.M. Scott, Joseph P. Macker, Gregory H.Cirincione "Internet Based Mobile Ad-hoc Networks" University of MarryLand, College Park, MD, Naval Research laboratory, Washington, DC, Army Research laboratory, Adelphi, MD.

[8] Luo Junhai, Xeu Liu, Ye Danxia (2007) "Multicast routing in MANET" University of science and technology, China, McGill University, Canada University of Science, China.

[9] Sivarammurthy C. "Mobile Ad-hoc Networks: Protocols \& Architecture " Pearson Edition

[10] Tripathy Akhilesh and Kumar Rakesh "A modified Energy Constrained Base Protocol for Mobile Ad-hoc Networks" Gorakhpur, India.

[11] Sharma Rohit and Sharma Sangeeta, "An Energy Efficient Approach Based On AODV" Lovely Professional University, India.

[12] Geier Jim (2004) “Wireless Networks First Step" Cisco Press 